\title{
Coding polymorphisms of bone morphogenetic protein 2 contribute to the development of childhood IgA nephropathy
}

\author{
JIN-SOON SUH ${ }^{1}$, WON-HO HAHN ${ }^{1}$, JONG SEOK LEE ${ }^{2}$, HAE JEONG PARK ${ }^{3}$, \\ MI-JA KIM ${ }^{3}$, SUNG WOOK KANG ${ }^{3}$, JOO-HO CHUNG ${ }^{3}$ and BYOUNG-SOO CHO ${ }^{1}$ \\ ${ }^{1}$ Department of Pediatrics, East West Kidney Disease Research Institute, ${ }^{2}$ Department of Emergency Medicine, and \\ ${ }^{3}$ Kohwang Medical Research Institute, School of Medicine, Kyung Hee University, Seoul 130-701, Republic of Korea
}

Received November 4, 2010; Accepted January 4, 2011

DOI: $10.3892 / \mathrm{etm} .2011 .195$

\begin{abstract}
Bone morphogenetic proteins (BMPs) are multifunctional growth factors belonging to the transforming growth factor $\beta$ (TGFB) superfamily and are important in both preservation of kidney function and resistance to injury. BMP2 is highly regulated in the kidney, and high affinity binding sites for BMP2 have been identified in kidney epithelial cells. BMP2 has been demonstrated to play various roles in the pathogenesis of renal diseases. However, the role of the BMP2 gene in glomerulonephritis has not been previously investigated. We aimed to evaluate the association of BMP2 gene polymorphisms with immunoglobulin A nephropathy (IgAN) in children. We evaluated 187 pediatric patients with biopsy-confimed IgAN and 262 healthy controls. Two coding single nucleotide polymorphisms (cSNPs) in the BMP2 gene [rs235768 (missense, Arg190Ser) and rs1049007 (synonymous, Ser87Ser)] were selected and genotyped by direct sequencing. Genotypes of rs1049007 were associated with childhood IgAN in the codominant model II (GG vs. AA) $[\mathrm{p}=0.02$; OR $(95 \% \mathrm{CI}), 0.16(0.04-0.70)]$ and in the recessive model [ $\mathrm{p}=0.0023$; OR $(95 \% \mathrm{CI}), 0.16(0.04-0.69)]$. We also found an association between rs235768 and IgAN in the codominant model II (TT vs. AA) [p=0.01; OR $(95 \%$ CI), 0.08 (0.01-0.57)] and in the recessive model $[\mathrm{p}=0.0002$; OR (95\% CI), 0.07 (0.01-0.55)]. After Bonferroni correction, these associations of rs235768 and rs1049007 with IgAN risk remained significant. In the haplotype analysis, the TG haplotype [p=0.01; OR (95\% CI), $6.76(1.55-29.50)$ in the dominant model] and AA haplotype [p=0.01; OR (95\% CI), 0.08 (0.01$0.59)$ in the recessive model] showed associations with IgAN.
\end{abstract}

Correspondence to: Dr Byoung-Soo Cho, Department of Pediatrics, East West Kidney Disease Research Institute, School of Medicine, Kyung Hee University, 1 Hoegi-dong, Dongdaemun-gu, Seoul 130-701, Republic of Korea

E-mail: bscho@dreamwiz.com

Key words: bone morphogenetic protein 2, polymorphism, immunoglobulin A nephropathy, children
The BMP2 gene may contribute to susceptibility to $\operatorname{Ig} A N$ in Korean children.

\section{Introduction}

Immunoglobulin A nephropathy ( $\operatorname{IgAN})$ is the most common form of primary glomerulonephritis (GN). Its hallmark is deposition of polymeric IgA1 in the glomerular mesangium accompanied by mesangial proliferative GN (1). The mechanisms involved in mesangial IgA deposition and the initiation of inflammatory glomerular injury remain unclear. The degree of histopathologic injury is extremely variable; this is reflected in the varied pace and severity of clinical presentation noted in this disease. Genetic factors contribute to the development of IgAN and the progression to renal failure, including the rate of $\operatorname{Ig} \mathrm{A}$ deposition, the phenotype of mesangial response and the risk of progressive renal failure (2).

Bone morphogenetic proteins (BMPs) are the largest group of members of the transforming growth factor $\beta$ (TGFB) superfamily and were originally identified as boneinducer proteins (3). In recent years, the importance of BMP signaling has been linked to the morphogenesis, cell proliferation and differentiation, apoptosis and patterning of various organs (4). In the kidney, several BMPs are expressed during organogenesis of the metanephric kidney, and in postnatal life BMPs have been shown to be important in both preservation of kidney function and resistance to injury (5).

BMP2 is a specific member of the BMPs and is highly regulated in the kidney (6). Moreover, high affinity binding sites for BMP2 have been identified in kidney epithelial cells (7). A few studies have suggested associations between BMP2 and renal diseases. In mesangial cells, BMP2 has been shown to inhibit platelet-derived growth factor (PDGF)-induced c-fos gene transcription and epidermal growth factor (EGF)-induced Elk transcription as well as DNA synthesis mediated by mitogen-activated protein kinase (MAPK) $(8,9)$. In podocytes, BMP2 induces reactive oxygen species via up-regulation of Id-1 expression, which subsequently inhibits cellular functions (10). Notably, together with BMP7, BMP2 has been demonstrated to have the same effect on renal fibrosis by promoting catabolism of type I TGFB receptors (6).

However, the association between IgAN and the BMP2 gene has not yet been reported. With regard to the associations 
between IgAN and BMPs, only BMP7 has been reported by Chan et al, who demonstrated that BMP7 has a beneficial effect on IgAN by ameliorating IgAN-derived polymeric IgA-induced tumor necrosis factor (TNF) (anti-inflammatory effect) and lymphotoxin $\alpha$ (TNF superfamily, member 1, LTA) synthesis (anti-fibrotic effect) in human mesangial cells (11).

In the present study, we investigated the association between IgAN and polymorphisms of the BMP2 gene.

\section{Materials and methods}

Subjects. We examined 187 Korean pediatric patients with biopsy-confirmed IgAN and 262 healthy controls. Almost all of the patients were identified through a urinary screening program at schools in Korea. Most of the patients showed no symptoms of renal disease and were thought to be at a relatively early stage of the disease. Patients that fulfilled one or more of the following criteria underwent a renal biopsy: i) unexplained prolonged isolated hematuria or proteinuria with a duration of $>12$ months; ii) concomitant hematuria and proteinuria with a duration of $>3$ months; iii) hematuria or proteinuria with decreased serum $\mathrm{C} 3$ and $\mathrm{C} 4$ levels; or iv) decreased renal function. Healthy controls were also recruited based on the findings of a routine screening. The screening included the completion of a questionnaire that included the following: symptoms and medical history, blood pressure, electrocardiography, abdominal sonography and laboratory tests, such as complete blood count, fasting glucose level, total cholesterol, triglyceride levels, high-density lipoprotein cholesterol levels, rheumatoid factor, hepatitis $\mathrm{B}$ and $\mathrm{C}$ viral markers, hemoglobin $\mathrm{A} 1 \mathrm{C}$, liver enzymes, blood urea nitrogen, creatinine, electrolytes and a urinalysis (protein, occult blood and glucose). Controls with any abnormal results were excluded. In this study, we did not age-match controls and patients. One of the confounding factors in genetic analysis is the presence of subclinical IgAN in supposedly normal control populations (2). IgAN is known to develop in young adults in their twenties. For this reason, healthy controls $>20$ years of age were needed to exclude the possibility of subclinical IgAN. Therefore, the presence of an age gap between the two groups appears unavoidable. This study was approved by the Ethics Review Committee of the Medical Research Institute, Kyung Hee University Medical Center, Seoul, Korea. Written informed consent was obtained from all of the subjects.

Patient subgroups. To determine the nature of the association between single nucleotide polymorphisms (SNPs) of BMP2 and the patient phenotype and histopathological findings, patients were divided into subgroups according to the following laboratory findings: i) the presence of proteinuria at the time of the initial renal biopsy (proteinuria $\leq 4$ or $>4 \mathrm{mg} / \mathrm{m}^{2} / \mathrm{h}$ ); ii) the presence of gross hematuria. Subgroups were also characterized on the basis of the following: iii) the presence of podocyte foot process effacement upon renal biopsy; iv) disease stage as assessed by pathological markers upon renal biopsy, such as interstitial fibrosis, tubular atrophy or global sclerosis (subjects who had one or more markers were placed in the advanced group and subjects without any of the pathological markers were placed in the early group); and v) the degree of mesangial
Table I. Demographic characteristics of the participating subjects.

\begin{tabular}{|c|c|c|}
\hline & IgAN & Control \\
\hline No. of subjects (n) & 187 & 262 \\
\hline Age $($ mean $\pm \mathrm{SD})$, years & $38.2 \pm 13.8$ & $12.4 \pm 4.5$ \\
\hline $\begin{array}{l}\text { Proteinuria }(\mathrm{n})^{\mathrm{a}} \\
>4 \mathrm{mg} / \mathrm{m}^{2} / \mathrm{h} \\
\leq 4 \mathrm{mg} / \mathrm{m}^{2} / \mathrm{h}\end{array}$ & $\begin{array}{l}88 \\
99\end{array}$ & \\
\hline $\begin{array}{l}\text { Gross hematuria }(\mathrm{n}) \\
+ \\
-\end{array}$ & $\begin{array}{r}35 \\
152\end{array}$ & \\
\hline $\begin{array}{l}\text { Podocyte foot process effacement (n) } \\
+ \\
-\end{array}$ & $\begin{array}{r}78 \\
109\end{array}$ & \\
\hline $\begin{array}{l}\text { Advanced pathological grading }(\mathrm{n})^{\mathrm{b}} \\
\text { Advanced } \\
\text { Mild }\end{array}$ & $\begin{array}{r}23 \\
164\end{array}$ & \\
\hline $\begin{array}{l}\text { Mesangial proliferation }(\mathrm{n})^{\mathrm{c}} \\
\text { Advanced } \\
\text { Mild }\end{array}$ & $\begin{array}{r}65 \\
122\end{array}$ & \\
\hline
\end{tabular}

aProteinuria refers to the amount of proteinuria at the time of renal biopsy. ${ }^{\text {bPatients }}$ were subgrouped into pathologically mild and advanced disease groups according to whether they had one or more of the three advanced pathologic markers (interstitial fibrosis, tubular atrophy or global sclerosis) at renal biopsy. ${ }^{\mathrm{c}}$ Mesangial proliferation was assessed according to the degree of mesangial proliferation: mild (normal to mild focal mesangial proliferation) vs. advanced (mild diffuse to severe diffuse mesangial proliferation).n, number of subjects; IgAN, immunoglobulin A nephropathy; SD, standard deviation.

proliferation (subjects with normal to mild focal mesangial proliferation were allocated to the mild group and those with mild diffuse to severe diffuse mesangial proliferation were allocated to the advanced group). All the demographic characteristics of the IgAN patients are documented in Table I.

SNP selection and genotyping. We searched for coding SNPs (cSNPs) in the BMP2 gene based on the results of database searches (http://www.ncbi.nlm.nih.gov/SNP, dbSNP BUILD 131). Among the SNPs in the coding regions, we excluded those with heterozygosity $<0.1$ (rs2273073, rs79417223, rs2273074, rs34183594 and rs11545591) or without a genotype frequency (rs13037675). Finally, rs1049007 (synonymous, Ser87Ser) and rs235768 (missense, Arg190Ser) were selected. The heterozygosities of rs1049007 and rs235768 were 0.397 and 0.382 , respectively.

DNA was isolated from peripheral blood samples using the Roche DNA Extraction kit (Roche, Indianapolis, IN, USA). SNP genotyping was performed by direct sequencing. Genomic DNA was amplified using primers specific for each cSNP of the BMP2 gene (Table II). The samples were sequenced using ABI PRISM 3730XL analyzer (PE Applied Biosystems, USA). Sequence data were analyzed using SeqManII software (DNAStar, Madison, WI, USA). 
Table II. Sequences of the primers used for each SNP in the BMP2 gene.

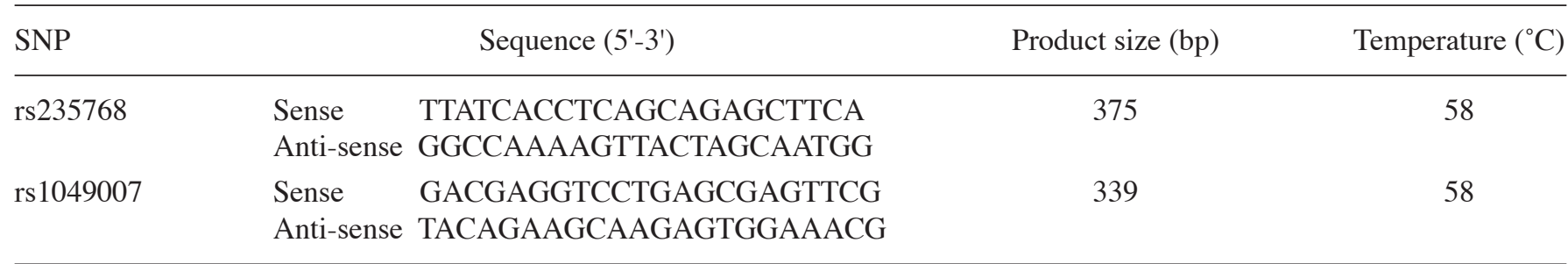

SNP, single nucleotide polymorphism; BMP2, bone morphogenetic protein 2; bp, base pair.

Table III. Logistic regression analysis of genotype and allelic frequencies of BMP2 gene polymorphisms in IgAN patients and controls.

\begin{tabular}{|c|c|c|c|c|c|c|}
\hline SNP & Type & Control, n (\%) & IgAN, n (\%) & Model & OR $(95 \% \mathrm{CI})$ & p-value \\
\hline rs235768 & Genotype & & & & & \\
\hline (missense & $\mathrm{TT}$ & $165(62.98)$ & $123(65.77)$ & Codominant I & $1.10(0.73-1.65)$ & 0.6500 \\
\hline \multirow[t]{6}{*}{ Arg190Ser) } & TA & $79(30.15)$ & $63(33.70)$ & Codominant II & $0.08(0.01-0.57)$ & 0.0100 \\
\hline & $\mathrm{AA}$ & $18(6.87)$ & $1 \quad(0.53)$ & Dominant & $0.91(0.61-1.35)$ & 0.6200 \\
\hline & & & & Recessive & 0.07 (0.01-0.55) & 0.0002 \\
\hline & Allele & & & & & \\
\hline & $\mathrm{T}$ & $409(78.05)$ & $309(82.62)$ & & $0.75(0.53-1.05)$ & 0.0900 \\
\hline & A & $115(21.95)$ & $65(17.38)$ & & & \\
\hline rs1049007 & Genotype & & & & & \\
\hline (synonymous & GG & $168(64.12)$ & 127 (67.91) & Codominant I & $1.02(0.68-0.04)$ & 0.9200 \\
\hline \multirow[t]{6}{*}{ Ser87Ser) } & GA & 77 (29.39) & $58(31.02)$ & Codominant II & $0.16(0.04-0.70)$ & 0.0200 \\
\hline & $\mathrm{AA}$ & 17 (6.49) & $2(1.07)$ & Dominant & $0.86(0.58-1.29)$ & 0.4700 \\
\hline & & & & Recessive & $0.16(0.04-0.69)$ & 0.0023 \\
\hline & Allele & & & & & \\
\hline & G & $413(78.82)$ & $312(83.42)$ & & $0.74(0.52-1.04)$ & 0.0800 \\
\hline & A & $111(21.18)$ & $62(16.58)$ & & & \\
\hline
\end{tabular}

p-values were obtained from logistic regression analysis with codominant, dominant and recessive models controlling gender as covariate. OR, 95\% CI and p-value of codominant model I and II were obtained by the comparison of respectively heterozygous and minor homozygous genotypes with the major homozygous genotype. Bold type indicates statistically significant values. SNP, single nucleotide polymorphism; BMP2, bone morphogenetic protein 2; IgAN, immunoglobulin A nephropathy; OR, odds ratio; CI, confidence interval; n, number of subjects .

Statistical analysis. For the case-control association study, all cSNPs in the cases and controls were assessed for HardyWeinberg equilibrium (HWE) using SNPstats software (Biostatistics and Bioinformatics Unit, Barcelona, Spain) (12). Logistic regression analysis was used to calculate the odds ratios (OR), 95\% confidence intervals (CIs) and p-values. For multiple logistic regression analyses to determine associations of single SNPs with IgAN, multiple inheritance models, including codominant 1 (major allele homozygotes vs. heterozygotes), codominant 2 (major allele homozygotes vs. minor allele homozygotes), dominant (major allele homozygotes vs. minor allele homozygotes plus heterozygotes) and recessive (major allele homozygotes plus heterozygotes vs. minor allele homozygotes) models were used. For comparisons between the patients with IgAN and healthy controls, the data were adjusted for gender only, and for comparisons among the IgAN subgroups, gender and age were controlled. Allelic frequencies of each cSNP were compared by Pearson's Chi-square test. A linkage disequilibrium (LD) block of polymorphisms was tested using Haploview version 4.1 (Broad Institute, Cambridge, MA, USA). SNPstats and HapAnalyzer Pro version 1.0 (http://www.hap.ngri.go.kr), and HelixTree (Golden Helix Inc., Bezemen, MT, USA) were used to analyze the association between SNPs and haplotypes. Statistical analyses were performed using the SPSS statistics 18.0 software package (SPSS Inc., Chicago, IL, USA), and a p-value of $<0.05$ was considered statistically significant. To avoid chance findings due to multiple testing, the Bonferroni correction was applied by lowering the significance levels to $p=0.025(p=0.05 / 2)$ for the two SNPs and for the two haplotypes.

\section{Results}

We investigated whether or not BMP2 gene polymorphisms are related to IgAN by genotyping two cSNPs in Korean children. The genetic distributions of all cSNPs in this study were in agreement with HWE ( $\mathrm{p}>0.05)$. 
Table IV. Haplotype analysis of BMP2 gene polymorphisms in the IgAN patients and controls.

\begin{tabular}{|c|c|c|c|c|c|c|c|c|}
\hline \multirow[t]{2}{*}{ Haplotype } & \multirow[t]{2}{*}{ Type } & \multicolumn{2}{|c|}{ Control } & \multicolumn{2}{|c|}{ IgAN } & \multirow[t]{2}{*}{ Model } & \multirow[t]{2}{*}{ OR $(95 \% \mathrm{CI})$} & \multirow[t]{2}{*}{ p-value } \\
\hline & & Frequency & $\mathrm{n}(\%)$ & Frequency & $\mathrm{n}(\%)$ & & & \\
\hline \multirow[t]{3}{*}{ TG } & $\mathrm{H} / \mathrm{H}$ & 0.78 & $164(62.36)$ & 0.83 & $123(66.13)$ & Codominant & $1.34(0.96-1.88)$ & 0.08 \\
\hline & $\mathrm{H} /-$ & & $81(30.80)$ & & $61(32.80)$ & Dominant & $6.76(1.55-29.5)$ & 0.01 \\
\hline & $-/-$ & & $18(6.84)$ & & $2(1.07)$ & Recessive & $1.18(0.80-1.75)$ & 0.41 \\
\hline \multirow[t]{3}{*}{ AA } & $\mathrm{H} / \mathrm{H}$ & 0.21 & $17 \quad(6.46)$ & 0.16 & $1(0.54)$ & Codominant & $0.72(0.51-1.02)$ & 0.07 \\
\hline & $\mathrm{H} /-$ & & $77(29.28)$ & & $58(31.18)$ & Dominant & $0.84(0.56-1.24)$ & 0.38 \\
\hline & $-/-$ & & $169(64.26)$ & & $127(68.28)$ & Recessive & $0.08(0.01-0.59)$ & 0.01 \\
\hline
\end{tabular}

Haplotype is comprised of rs235768 and rs 1049007 in BMP2. p-values were obtained from logistic regression analysis with codominant, dominant and recessive models. Bold type indicates statistically significant values. SNP, single nucleotide polymorphism; BMP2, bone morphogenetic protein 2; IgAN, immunoglobulin A nephropathy; OR, odds ratio; CI, confidence interval; n, number of subjects.

As shown in Table III, the genotyping data revealed that two cSNPs in the coding region were significantly associated with $\operatorname{IgAN}$. The rs 235768 was associated with $\operatorname{IgAN}$ in the codominant model II [p=0.01; OR $(95 \% \mathrm{CI}), 0.08(0.01$ $0.57)]$ and in the recessive model $[\mathrm{p}=0.0002$; OR $(95 \% \mathrm{CI})$, 0.07 (0.01-0.55)]. A significant association was also observed between IgAN and rs1049007 in the codominant model II $[\mathrm{p}=0.02$; OR $(95 \% \mathrm{CI}), 0.16(0.04-0.70)]$ and in the recessive model $[\mathrm{p}=0.0023$; OR $(95 \% \mathrm{CI}), 0.16(0.04-0.69)]$. These results remained significant after Bonferroni correction $(p<0.025)$. However, allelic frequencies were not associated with IgAN (Table III). Comparisons of the subgroups did not show any significant associations with the cSNPs (data not shown).

Through LD block analysis using the Gabriel method (13), we found that an LD block was made between two cSNPs (Fig. 1). In haplotype analysis, haplotypes TG [p=0.01; OR (95\% CI), $6.76(1.55-29.50)$ in the dominant model] and AA [p=0.01; OR (95\% CI), 0.08 (0.01-0.59) in the recessive model] were associated with IgAN (Table IV). The significant association of haplotypes with IgAN remained after Bonferroni correction $(\mathrm{p}<0.025)$.

We calculated the sample power to verify our data, using a genetic power calculator (http://pngu.mgh.harvard. edu/ purcell/gpc/cc2.html). In our case-control study, sample powers for the SNPs were 0.825 (rs235768, number of cases for $70 \%$ power $=175)$ and 0.814 (rs1049007, number of cases for $70 \%$ power $=180)$, respectively $(\alpha=0.05$, genotype relative risk 2-fold). Therefore, our case-control study was sufficiently powered for rs 235768 and rs1049007 to determine a positive association.

\section{Discussion}

Bone morphogenetic proteins are multi-functional growth factors and belong to the TGFB superfamily (3). Their functions include the regulation of embryonic development and cellular homeostasis, such as the regulation of proliferation, differentiation, apoptosis and remodeling of the extracellular matrix. Dysregulation of BMPs has been documented in several types of diseases, including cancer, diabetes, fibrosis and renal, auto-immune and cardiovascular diseases (4,14-17).

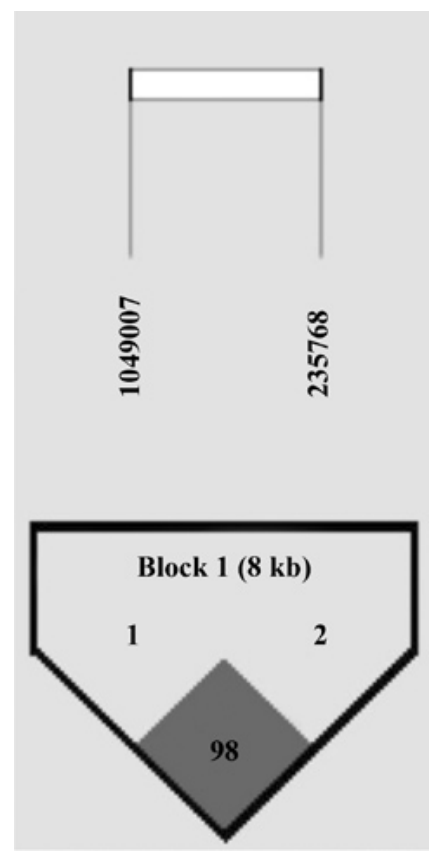

Figure 1. Linkage disequilibrium (LD) block between two single nucleotide polymorphisms of the bone morphogenetic protein 2 gene. Black shading indicates strong LD.

BMP2 was originally identified as an essential inducer for bone development and repair (18). Clinically, BMP2 has been used for various therapeutic interventions, such as bone defects, non-union fracture, spinal fusion, osteoporosis and root canal surgery (3). Apart from bone disease, BMP2 has been shown to be associated with invasion and metastasis of several tumor cell lines $(15,19,20)$ and is used as a prognostic marker for specific tumors (21). Moreover, it is involved in vascular physiology as a proatherogenic mediator in the arterial wall (4). However, limited data have been reported for the association between BMP2 and renal diseases $(6,22,23)$.

In the present study, the genotypes of rs235768 (missense, Arg190Ser) and rs1049007 (synonymous, Ser87Ser) were associated with childhood IgAN in a case-control analysis. In addition, the TG and AA haplotypes consisting of rs 235768 and rs1049007 were associated with IgAN. However, the 
mechanisms by which they are involved in the development of IgAN are not yet known.

BMPs exert their biologic actions via transmembrane serine/threonine kinase receptors. Binding of BMP to its receptor stimulates oligomerization of the receptor complex. According to the different oligomerization modes, BMP2 activates distinct signaling pathways, including the classical Smad pathway and non-Smad pathways, such as MAPK signaling through transcriptional effects, and also has non-transcriptional effects, such as cytoskeletal rearrangements (24). Previous in vitro studies have demonstrated that BMP2 inhibits MAPK-dependent gene transcription and DNA synthesis in mesangial cells $(8,9)$. Further studies that discriminate between the distinct pathways in the kidneys of IgAN patients with such polymorphisms may aid in the understanding of IgAN pathogenesis and present the possibility of individualized gene or cell therapy (4).

Additionally, we analyzed the association between BMP2 gene polymorphisms and disease progression using clinical and histological parameters. However, neither of the two SNPs showed an association with any of the parameters for disease progression. One possible reason is that our patients were enrolled through urine screening tests; therefore, most of them showed no symptoms and had relatively mild GN. Further studies with subjects in various stages of $\operatorname{Ig} \mathrm{AN}$ are required to evaluate the association with disease progression.

In conclusion, this study is the first to evaluate the association of BMP2 gene polymorphisms with childhood IgAN. The rs235768 and rs1049007 cSNPs of the BMP2 gene may be associated with $\mathrm{IgAN}$ in the Korean population.

\section{Acknowledgements}

This study was supported by the Program at Kyung Hee University for the Young Researcher of Medical Science in 2009 (KHU-20091456).

\section{References}

1. Barratt $\mathbf{J}$ and Feehally J: IgA nephropathy. J Am Soc Nephrol 16: 2088-2097, 2005.

2. Barratt J, Smith AC, Molyneux $\mathrm{K}$ and Feehally J: Immunopathogenesis of IgAN. Semin Immunopathol 29: 427-443, 2007.

3. Chen D, Zhao M and Mundy GR: Bone morphogenetic proteins. Growth Factors 22: 233-241, 2004.

4. Maciel TT, Kempf H and Campos AH: Targeting bone morphogenetic protein signaling on renal and vascular diseases. Curr Opin Nephrol Hypertens 19: 26-31, 2010.

5. Simic P and Vukicevic S: Bone morphogenetic proteins in development and homeostasis of kidney. Cytokine Growth Factor Rev 16: 299-308, 2005

6. Yang YL, Liu YS, Chuang LY, et al: Bone morphogenetic protein-2 antagonizes renal interstitial fibrosis by promoting catabolism of type I transforming growth factor-beta receptors. Endocrinology 150: 727-740, 2009.
7. Iwasaki S, Tsuruoka N, Hattori A, Sato M, Tsujimoto M and Kohno M: Distribution and characterization of specific cellular binding proteins for bone morphogenetic protein-2. J Biol Chem 270: 5476-5482, 1995

8. Ghosh Choudhury G, Kim YS, Simon M, et al: Bone morphogenetic protein 2 inhibits platelet-derived growth factor-induced c-fos gene transcription and DNA synthesis in mesangial cells. Involvement of mitogen-activated protein kinase. J Biol Chem 274: 10897-10902, 1999

9. Ghosh Choudhury G, Jin DC, Kim Y, Celeste A, Ghosh-Choudhury $\mathrm{N}$ and Abboud HE: Bone morphogenetic protein-2 inhibits MAPK-dependent Elk-1 transactivation and DNA synthesis induced by EGF in mesangial cells. Biochem Biophys Res Commun 258: 490-496, 1999.

10. Pache G, Schafer C, Wiesemann S, et al: Upregulation of Id-1 via BMP-2 receptors induces reactive oxygen species in podocytes. Am J Physiol Renal Physiol 291: F654-F662, 2006.

11. Chan WL, Leung JC, Chan LY, Tam KY, Tang SC and Lai KN: BMP-7 protects mesangial cells from injury by polymeric IgA. Kidney Int 74: 1026-1039, 2008.

12. Sole X, Guino E, Valls J, Iniesta R and Moreno V: SNPStats: a web tool for the analysis of association studies. Bioinformatics 22: 1928-1929, 2006.

13. Gabriel SB, Schaffner SF, Nguyen H, et al: The structure of haplotype blocks in the human genome. Science 296: 2225-2229, 2002.

14. Nguyen TQ, Roestenberg P, van Nieuwenhoven FA, et al: CTGF inhibits BMP-7 signaling in diabetic nephropathy. J Am Soc Nephrol 19: 2098-2107, 2008

15. Park Y, Kim JW, Kim DS, et al: The bone morphogenesis protein-2 (BMP-2) is associated with progression to metastatic disease in gastric cancer. Cancer Res Treat 40: 127-132, 2008.

16. Ara J, See J, Mamontov P, et al: Bone morphogenetic proteins 4,6 , and 7 are up-regulated in mouse spinal cord during experimental autoimmune encephalomyelitis. J Neurosci Res 86: $125-135,2008$

17. Weiskirchen R, Meurer SK, Gressner OA, Herrmann J, Borkham-Kamphorst E and Gressner AM: BMP-7 as antagonist of organ fibrosis. Front Biosci 14: 4992-5012, 2009.

18. Rosen V: BMP2 signaling in bone development and repair. Cytokine Growth Factor Rev 20: 475-480, 2009.

19. Langenfeld EM, Calvano SE, Abou-Nukta F,Lowry SF, Amenta P and Langenfeld $\mathrm{J}$ : The mature bone morphogenetic protein-2 is aberrantly expressed in non-small cell lung carcinomas and stimulates tumor growth of A549 cells. Carcinogenesis 24: 1445-1454, 2003.

20. Kleeff J, Maruyama H, Ishiwata $\mathrm{T}$, et al: Bone morphogenetic protein 2 exerts diverse effects on cell growth in vitro and is expressed in human pancreatic cancer in vivo. Gastroenterology 116: 1202-1216, 1999.

21. Liu C, Tian G, Tu Y, Fu J, Lan C and Wu N: Expression pattern and clinical prognostic relevance of bone morphogenetic protein-2 in human gliomas. Jpn J Clin Oncol 39: 625-631, 2009.

22. Baelde HJ, Eikmans M, Doran PP, Lappin DW, de Heer E and Bruijn JA: Gene expression profiling in glomeruli from human kidneys with diabetic nephropathy. Am J Kidney Dis 43: 636-650, 2004.

23. Dalfino G, Simone S, Porreca S, et al: Bone morphogenetic protein-2 may represent the molecular link between oxidative stress and vascular stiffness in chronic kidney disease. Atherosclerosis 211: 418-423, 2010.

24. Sieber C, Kopf J, Hiepen C and Knaus P: Recent advances in BMP receptor signaling. Cytokine Growth Factor Rev 20: 343-355, 2009. 